\title{
EXPLICIT CRITERIA FOR THE OSCILLATION OF DIFFERENTIAL EQUATIONS WITH SEVERAL ARGUMENTS
}

\author{
GEORGE E. CHATZARAKIS ${ }^{1}$ AND IRENA JADLOVSKÁ ${ }^{2}$ \\ ${ }^{1}$ Department of Electrical and Electronic Engineering Educators \\ School of Pedagogical and Technological Education (ASPETE) \\ 14121, N. Heraklio, Athens, GREECE \\ ${ }^{2}$ Departmens of Mathematics and Theoretical Informatics \\ Faculty of Electrical Engineering and Informatics \\ Technical University of Košice \\ Letná 9, 04200 Košice, SLOVAKIA
}

\begin{abstract}
In this paper we are concerned with the oscillations of differential equations with several non-monotone deviating arguments and nonnegative coefficients. We present new sufficient conditions, involving limsup and liminf, for the oscillation of all solutions which essentially improve several known criteria existing in the literature. We illustrate the results and the improvement over other known oscillation criteria by examples, numerically solved in MATLAB.
\end{abstract}

AMS Subject Classification: $34 \mathrm{~K} 11,34 \mathrm{~K} 06$

Key Words: differential equation, non-monotone argument, oscillatory solution, nonoscillatory solution

Received: October 3, 2018; Revised: January 28, 2019;

Published (online): February 18, $2019 \quad$ doi: $10.12732 /$ dsa.v28i2.1

Dynamic Publishers, Inc., Acad. Publishers, Ltd. https://acadsol.eu/dsa

\section{INTRODUCTION}

Consider the first-order linear differential equation with several variable deviating arguments of either delay (DDE)

$$
x^{\prime}(t)+\sum_{i=1}^{m} p_{i}(t) x\left(\tau_{i}(t)\right)=0, \quad \forall t \geq t_{0},
$$

or advanced type (ADE)

$$
x^{\prime}(t)-\sum_{i=1}^{m} q_{i}(t) x\left(\sigma_{i}(t)\right)=0, \quad t \geq t_{0},
$$


where $p_{i}, q_{i}, 1 \leq i \leq m$, are nonnegative real functions, and $\tau_{i}, \sigma_{i}, 1 \leq i \leq m$, are positive real functions such that

$$
\tau_{i}(t)<t, \quad t \geq t_{0} \quad \text { and } \quad \lim _{t \rightarrow \infty} \tau_{i}(t)=\infty, \quad 1 \leq i \leq m
$$

and

$$
\sigma_{i}(t)>t, \quad t \geq t_{0}, \quad 1 \leq i \leq m,
$$

respectively.

A solution of $(E)$ of $\left(E^{\prime}\right)$ is an absolutely continuous on $\left[t_{0}, \infty\right)$ function satisfying $(E)$ or $\left(E^{\prime}\right)$ for almost all $t \geq t_{0}$.

A solution of $(E)$ or $\left(E^{\prime}\right)$ is oscillatory, if it is neither eventually positive nor eventually negative. If there exists an eventually positive or an eventually negative solution, the equation is nonoscillatory. An equation is oscillatory if all its solutions oscillate.

The problem of establishing sufficient conditions for the oscillation of all solutions of equations $(E)$ or $\left(E^{\prime}\right)$ has been the subject of many investigations. The reader is referred to $[1-4,6-18,20-24]$ and the references cited therein. Most of these papers concern the special case where the arguments are nondecreasing, while a small number of these papers are dealing with the general case where the arguments are not necessarily monotone. See, for example, $[1-4,8,13]$ and the references cited therein. Apart from the pure mathematical interest, the importance of considering non-monotone arguments is justified by the fact that they approximate the natural phenomena described by equations of the type $(E)$ or $\left(E^{\prime}\right)$. That is because there are always natural disturbances (e.g. noise in communication systems) that affect all the parameters of the equation and therefore the fair (from a mathematical point of view) monotone arguments become non-monotone almost always.

Throughout this paper, we are going to use the following notation:

$$
\alpha:=\liminf _{t \rightarrow \infty} \int_{\tau(t)}^{t} \sum_{i=1}^{m} p_{i}(s) d s, \quad \beta:=\liminf _{t \rightarrow \infty} \int_{t}^{\sigma(t)} \sum_{i=1}^{m} q_{i}(s) d s
$$

and

$$
D(\omega):= \begin{cases}0, & \text { if } \omega>1 / e \\ \frac{1-\omega-\sqrt{1-2 \omega-\omega^{2}}}{2}, & \text { if } \omega \in[0,1 / e] .\end{cases}
$$

\subsection{DDES}

By Remark 2.7.3 in [19], it is clear that if $\tau_{i}(t), 1 \leq i \leq m$ are nondecreasing and

$$
\limsup _{t \rightarrow \infty} \int_{\tau(t)}^{t} \sum_{i=1}^{m} p_{i}(s) d s>1
$$


where $\tau(t)=\max _{1 \leq i \leq m}\left\{\tau_{i}(t)\right\}$, then all solutions of $(E)$ oscillate. This result is similar to Theorem 2.1.3 [19] which is a special case of Ladas, Lakshmikantham and Papadakis's result [16].

In 1978 Ladde [18] and in 1982 Ladas and Stavroulakis [17] proved that if

$$
\liminf _{t \rightarrow \infty} \int_{\tau(t)}^{t} \sum_{i=1}^{m} p_{i}(s) d s>\frac{1}{e}
$$

then all solutions of $(E)$ oscillate.

In 1984, Hunt and Yorke [9] proved that if $t-\tau_{i}(t) \leq \tau_{0}, 1 \leq i \leq m$, and

$$
\liminf _{t \rightarrow \infty} \sum_{i=1}^{m} p_{i}(t)\left(t-\tau_{i}(t)\right)>\frac{1}{e}
$$

then all solutions of $(E)$ oscillate.

Assume that $\tau_{i}(t), 1 \leq i \leq m$ are not necessarily monotone. Set

$$
h_{i}(t)=\sup _{t_{0} \leq s \leq t} \tau_{i}(s), \quad t \geq t_{0} \quad \text { and } \quad h(t)=\max _{1 \leq i \leq m} h_{i}(t), \quad t \geq t_{0}
$$

and

$$
\begin{aligned}
a_{1}(t, s) & :=\exp \left\{\int_{s}^{t} \sum_{i=1}^{m} p_{i}(\zeta) d \zeta\right\} \\
a_{r+1}(t, s) & :=\exp \left\{\int_{s}^{t} \sum_{i=1}^{m} p_{i}(\zeta) a_{r}\left(\zeta, \tau_{i}(\zeta)\right) d \zeta\right\} .
\end{aligned}
$$

Clearly, $h_{i}(t), h(t)$ are nondecreasing and $\tau_{i}(t) \leq h_{i}(t) \leq h(t)<t$ for all $t \geq t_{0}$.

In 2016, Braverman, Chatzarakis and Stavroulakis [1] proved that if for some $r \in \mathbb{N}$

$$
\limsup _{t \rightarrow \infty} \int_{h(t)}^{t} \sum_{i=1}^{m} p_{i}(\zeta) a_{r}\left(h(t), \tau_{i}(\zeta)\right) d \zeta>1
$$

or

$$
\limsup _{t \rightarrow \infty} \int_{h(t)}^{t} \sum_{i=1}^{m} p_{i}(\zeta) a_{r}\left(h(t), \tau_{i}(\zeta)\right) d \zeta>1-D(\alpha),
$$

or

$$
\liminf _{t \rightarrow \infty} \int_{h(t)}^{t} \sum_{i=1}^{m} p_{i}(\zeta) a_{r}\left(h(t), \tau_{i}(\zeta)\right) d \zeta>\frac{1}{e},
$$

then all solutions of $(E)$ oscillate.

In 2017, Chatzarakis and Péics [3] proved that if

$$
\limsup _{t \rightarrow \infty} \int_{h(t)}^{t} \sum_{i=1}^{m} p_{i}(\zeta) a_{r}\left(h(\zeta), \tau_{i}(\zeta)\right) d \zeta>\frac{1+\ln \lambda_{0}}{\lambda_{0}}-D(\alpha),
$$

where $\lambda_{0}$ is the smaller root of the transcendental equation $e^{\alpha \lambda}=\lambda$, then all solutions of $(E)$ oscillate. 
In the same year, Chatzarakis [4] proved that if

$$
\bar{P}_{j}(t)=\bar{P}(t)\left[1+\int_{\tau(t)}^{t} \bar{P}(s) \exp \left(\int_{\tau(s)}^{t} \bar{P}(u) \exp \left(\int_{\tau(u)}^{u} \bar{P}_{j-1}(\xi) d \xi\right) d u\right) d s\right]
$$

with $\bar{P}_{0}(t)=\bar{P}(t)=\sum_{i=1}^{m} p_{i}(t)$, then for some $j \in \mathbb{N}$ either one of the conditions

$$
\begin{gathered}
\limsup _{t \rightarrow \infty} \int_{h(t)}^{t} \bar{P}(s) \exp \left(\int_{\tau(s)}^{h(t)} \bar{P}_{j}(u) d u\right) d s>1, \\
\limsup _{t \rightarrow \infty} \int_{h(t)}^{t} \bar{P}(s) \exp \left(\int_{\tau(s)}^{h(t)} \bar{P}_{j}(u) d u\right) d s>1-D(\alpha), \\
\limsup _{t \rightarrow \infty} \int_{h(t)}^{t} \bar{P}(s) \exp \left(\int_{\tau(s)}^{h(s)} \bar{P}_{j}(u) d u\right) d s>\frac{1+\ln \lambda_{0}}{\lambda_{0}}-D(\alpha), \\
\limsup _{t \rightarrow \infty} \int_{h(t)}^{t} \bar{P}(s) \exp \left(\int_{\tau(s)}^{t} \bar{P}_{j}(u) d u\right) d s>\frac{1}{D(\alpha)}
\end{gathered}
$$

and

$$
\liminf _{t \rightarrow \infty} \int_{h(t)}^{t} \bar{P}(s) \exp \left(\int_{\tau(s)}^{h(s)} \bar{P}_{j}(u) d u\right) d s>\frac{1}{e},
$$

implies that all solutions of $(E)$ are oscillatory.

\subsection{ADES}

For Eq. $\left(E^{\prime}\right)$, the dual condition of $(1.3)$ is

$$
\limsup _{t \rightarrow \infty} \int_{t}^{\sigma(t)} \sum_{i=1}^{m} q_{i}(s) d s>1
$$

where $\sigma_{i}(t), 1 \leq i \leq m$ are nondecreasing and $\sigma(t)=\min _{1 \leq i \leq m}\left\{\sigma_{i}(t)\right\}$, see [19], paragraph 2.7 .

In 1978 Ladde [18] and in 1982 Ladas and Stavroulakis [17] proved that if

$$
\liminf _{t \rightarrow \infty} \int_{t}^{\sigma(t)} \sum_{i=1}^{m} q_{i}(s) d s>\frac{1}{e}
$$

then all solutions of $\left(E^{\prime}\right)$ oscillate.

In 1990, Zhou [24] proved that if $\sigma_{i}(t)-t \leq \sigma_{0}, 1 \leq i \leq m$, and

$$
\liminf _{t \rightarrow \infty} \sum_{i=1}^{m} q_{i}(t)\left(\sigma_{i}(t)-t\right)>\frac{1}{e}
$$

then all solutions of $\left(E^{\prime}\right)$ oscillate. (See also [5, Corollary 2.6.12]) 
Assume that $\sigma_{i}(t), 1 \leq i \leq m$ are not necessarily monotone. Set

$$
\rho_{i}(t)=\inf _{s \geq t} \sigma_{i}(s), \quad t \geq t_{0} \quad \text { and } \quad \rho(t)=\min _{1 \leq i \leq m} \rho_{i}(t), \quad t \geq t_{0}
$$

and

$$
\begin{aligned}
b_{1}(t, s) & :=\exp \left\{\int_{t}^{s} \sum_{i=1}^{m} q_{i}(\zeta) d \zeta\right\} \\
b_{r+1}(t, s) & :=\exp \left\{\int_{t}^{s} \sum_{i=1}^{m} q_{i}(\zeta) b_{r}\left(t, \sigma_{i}(\zeta)\right) d \zeta\right\} .
\end{aligned}
$$

Clearly, $\rho_{i}(t), \rho(t)$ are nondecreasing and $\sigma_{i}(t) \geq \rho_{i}(t) \geq \rho(t)>t$ for all $t \geq t_{0}$.

In 2016, Braverman, Chatzarakis and Stavroulakis [1] proved that if for some $r \in \mathbb{N}$

$$
\limsup _{t \rightarrow \infty} \int_{t}^{\rho(t)} \sum_{i=1}^{m} q_{i}(\zeta) b_{r}\left(\rho(t), \sigma_{i}(\zeta)\right) d \zeta>1
$$

or

$$
\limsup _{t \rightarrow \infty} \int_{t}^{\rho(t)} \sum_{i=1}^{m} q_{i}(\zeta) b_{r}\left(\rho(t), \sigma_{i}(\zeta)\right) d \zeta>1-D(\beta)
$$

or

$$
\liminf _{t \rightarrow \infty} \int_{t}^{\rho(t)} \sum_{i=1}^{m} q_{i}(\zeta) b_{r}\left(\rho(t), \sigma_{i}(\zeta)\right) d \zeta>\frac{1}{e},
$$

then all solutions of $\left(E^{\prime}\right)$ oscillate.

In 2017, Chatzarakis [4] proved that if

$$
\bar{Q}_{j}(t)=\bar{Q}(t)\left[1+\int_{t}^{\sigma(t)} \bar{Q}(s) \exp \left(\int_{t}^{\sigma(s)} \bar{Q}(u) \exp \left(\int_{u}^{\sigma(u)} \bar{Q}_{j-1}(\xi) d \xi\right) d u\right) d s\right]
$$

with $\bar{Q}_{0}(t)=\bar{Q}(t)=\sum_{i=1}^{m} q_{i}(t)$, then, for some $j \in \mathbb{N}$ either one of the conditions

$$
\begin{gathered}
\limsup _{t \rightarrow \infty} \int_{t}^{\rho(t)} \bar{Q}(s) \exp \left(\int_{\rho(t)}^{\sigma(s)} \bar{Q}_{j}(u) d u\right) d s>1, \\
\limsup _{t \rightarrow \infty} \int_{t}^{\rho(t)} \bar{Q}(s) \exp \left(\int_{\rho(t)}^{\sigma(s)} \bar{Q}_{j}(u) d u\right) d s>1-D(\beta), \\
\limsup _{t \rightarrow \infty} \int_{t}^{\rho(t)} \bar{Q}(s) \exp \left(\int_{t}^{\sigma(s)} \bar{Q}_{j}(u) d u\right) d s>\frac{1}{D(\beta)}, \\
\limsup _{t \rightarrow \infty} \int_{t}^{\rho(t)} \bar{Q}(s) \exp \left(\int_{\rho(s)}^{\sigma(s)} \bar{Q}_{j}(u) d u\right) d s>\frac{1+\ln \lambda_{0}}{\lambda_{0}}-D(\beta)
\end{gathered}
$$

and

$$
\liminf _{t \rightarrow \infty} \int_{t}^{\rho(t)} \bar{Q}(s) \exp \left(\int_{\rho(s)}^{\sigma(s)} \bar{Q}_{j}(u) d u\right) d s>\frac{1}{e}
$$


implies that all solutions of $\left(E^{\prime}\right)$ are oscillatory.

The purpose of this paper is to derive sufficient conditions for all solutions of $(E)$ and $\left(E^{\prime}\right)$ to be oscillatory when the arguments are not necessarily monotone. Our results essentially improve several known criteria existing in the literature.

\section{MAIN RESULTS}

\subsection{DDES}

Based on an iterative technique, we further study $(E)$ and derive new sufficient oscillation conditions, involving limsup and liminf, which essentially improve several results in the literature.

We now cite three lemmas which will be used in the proof of our next results. The proofs of them are similar to the proofs of Lemmas 2.1.1, 2.1.3 and 2.1.2 in [5], respectively.

Lemma 1. Assume that $h(t)$ is defined by (1.6). Then

$$
\liminf _{t \rightarrow \infty} \int_{\tau(t)}^{t} \sum_{i=1}^{m} p_{i}(s) d s=\liminf _{t \rightarrow \infty} \int_{h(t)}^{t} \sum_{i=1}^{m} p_{i}(s) d s .
$$

Lemma 2. Assume that $x$ is an eventually positive solution of $(E)$ and $h(t)$ is defined by (1.6). Then

$$
\liminf _{t \rightarrow \infty} \frac{x(t)}{x(h(t))} \geq D(\alpha)
$$

Lemma 3. Assume that $x$ is an eventually positive solution of $(E)$ and $h(t)$ is defined by (1.6). Then

$$
\liminf _{t \rightarrow \infty} \frac{x(h(t))}{x(t)} \geq \lambda_{0},
$$

where $\lambda_{0}$ is the smaller root of the transcendental equation $\lambda=e^{\alpha \lambda}$.

Based on the above lemmas, we establish the following theorems.

Theorem 4. Assume that $h(t)$ is defined by (1.6) and for some $\ell \in \mathbb{N}$

$$
\limsup _{t \rightarrow \infty} \int_{h(t)}^{t} P(s) \exp \left(\int_{\tau(s)}^{h(t)} P(u) \exp \left(\int_{\tau(u)}^{u} G_{\ell}(\xi) d \xi\right) d u\right) d s>1,
$$

where

$$
G_{\ell}(t)=P(t)\left[1+\int_{\tau(t)}^{t} P(s) \exp \left(\int_{\tau(s)}^{t} P(u) \exp \left(\int_{\tau(u)}^{u} G_{\ell-1}(\xi) d \xi\right) d u\right) d s\right]
$$


with $G_{0}(t)=P(t)\left[1+\int_{\tau(t)}^{t} P(s) \exp \left(\lambda_{0} \int_{\tau(s)}^{t} P(u) d u\right) d s\right], P(t)=\sum_{i=1}^{m} p_{i}(t)$ and $\lambda_{0}$ is the smaller root of the transcendental equation $\lambda=e^{\alpha \lambda}$. Then all solutions of (E) are oscillatory.

Proof. Assume, for the sake of contradiction, that there exists a nonoscillatory solution $x(t)$ of $(E)$. Since $-x(t)$ is also a solution of $(E)$, we can confine our discussion only to the case where the solution $x(t)$ is eventually positive. Then there exists a $t_{1}>t_{0}$ such that $x(t)$ and $x\left(\tau_{i}(t)\right)>0$ for all $t \geq t_{1}$. Thus, from $(E)$ we have

$$
x^{\prime}(t)=-\sum_{i=1}^{m} p_{i}(t) x\left(\tau_{i}(t)\right) \leq 0 \text { for all } t \geq t_{1},
$$

which means that $x(t)$ is an eventually nonincreasing function of positive numbers.

Now we divide $(E)$ by $x(t)>0$ and integrate on $[s, t]$, so

$$
\int_{s}^{t} \frac{x^{\prime}(u)}{x(u)} d u=-\int_{s}^{t} \sum_{i=1}^{m} p_{i}(u) \frac{x\left(\tau_{i}(u)\right)}{x(u)} d u,
$$

or

$$
\int_{s}^{t} \frac{x^{\prime}(u)}{x(u)} d u \leq-\int_{s}^{t}\left(\sum_{i=1}^{m} p_{i}(u)\right) \frac{x(\tau(u))}{x(u)} d u,
$$

or

$$
\int_{s}^{t} \frac{x^{\prime}(u)}{x(u)} d u \leq-\int_{s}^{t} P(u) \frac{x(\tau(u))}{x(u)} d u
$$

Therefore

$$
\ln \frac{x(t)}{x(s)} \leq-\int_{s}^{t} P(u) \frac{x(\tau(u))}{x(u)} d u
$$

i.e.,

$$
x(s) \geq x(t) \exp \left(\int_{s}^{t} P(u) \frac{x(\tau(u))}{x(u)} d u\right) .
$$

Since $\tau(s)<s \leq t,(2.6)$ gives

$$
x(\tau(s)) \geq x(t) \exp \left(\int_{\tau(s)}^{t} P(u) \frac{x(\tau(u))}{x(u)} d u\right) .
$$

Integrating $(E)$ from $\tau(t)$ to $t$, we have

$$
x(t)-x(\tau(t))+\int_{\tau(t)}^{t} \sum_{i=1}^{m} p_{i}(s) x\left(\tau_{i}(s)\right) d s=0
$$

or

$$
x(t)-x(\tau(t))+\int_{\tau(t)}^{t} P(s) x(\tau(s)) d s \leq 0 .
$$

Combining (2.7) and (2.8), we have

$$
x(t)-x(\tau(t))+x(t) \int_{\tau(t)}^{t} P(s) \exp \left(\int_{\tau(s)}^{t} P(u) \frac{x(\tau(u))}{x(u)} d u\right) d s \leq 0 .
$$


Multiplying the last inequality by $P(t)$, we take

$$
P(t) x(t)-P(t) x(\tau(t))+P(t) x(t) \int_{\tau(t)}^{t} P(s) \exp \left(\int_{\tau(s)}^{t} P(u) \frac{x(\tau(u))}{x(u)} d u\right) d s \leq 0 .
$$

Furthermore,

$$
x^{\prime}(t)=-\sum_{i=1}^{m} p_{i}(t) x\left(\tau_{i}(t)\right) \leq-x(\tau(t)) \sum_{i=1}^{m} p_{i}(t)=-P(t) x(\tau(t)) .
$$

Combining the inequalities (2.9) and (2.10), we have

$$
x^{\prime}(t)+P(t) x(t)+P(t) x(t) \int_{\tau(t)}^{t} P(s) \exp \left(\int_{\tau(s)}^{t} P(u) \frac{x(\tau(u))}{x(u)} d u\right) d s \leq 0 .
$$

Since $\tau(u) \leq h(u)$, clearly

$$
x^{\prime}(t)+P(t) x(t)+P(t) x(t) \int_{\tau(t)}^{t} P(s) \exp \left(\int_{\tau(s)}^{t} P(u) \frac{x(h(u))}{x(u)} d u\right) d s \leq 0 .
$$

Taking into account the fact that (2.3) of Lemma 3 is satisfied, the last inequality becomes

$$
x^{\prime}(t)+P(t) x(t)+P(t) x(t) \int_{\tau(t)}^{t} P(s) \exp \left(\left(\lambda_{0}-\epsilon\right) \int_{\tau(s)}^{t} P(u) d u\right) d s \leq 0 .
$$

Thus

$$
x^{\prime}(t)+P(t)\left[1+\int_{\tau(t)}^{t} P(s) \exp \left(\left(\lambda_{0}-\epsilon\right) \int_{\tau(s)}^{t} P(u) d u\right) d s\right] x(t) \leq 0,
$$

or

$$
x^{\prime}(t)+G_{0}(t, \epsilon) x(t) \leq 0
$$

with

$$
G_{0}(t, \epsilon)=P(t)\left[1+\int_{\tau(t)}^{t} P(s) \exp \left(\left(\lambda_{0}-\epsilon\right) \int_{\tau(s)}^{t} P(u) d u\right) d s\right] .
$$

Applying the Grönwall inequality in (2.11), we obtain

$$
x(s) \geq x(t) \exp \left(\int_{s}^{t} G_{0}(\xi, \epsilon) d \xi\right), \quad t \geq s .
$$

Thus

$$
x(\tau(u)) \geq x(u) \exp \left(\int_{\tau(u)}^{u} G_{0}(\xi, \epsilon) d \xi\right) .
$$

Now we divide $(E)$ by $x(t)>0$ and integrate on $[s, t]$, so

$$
-\int_{s}^{t} \frac{x^{\prime}(u)}{x(u)} d u=\int_{s}^{t} \sum_{i=1}^{m} p_{i}(u) \frac{x\left(\tau_{i}(u)\right)}{x(u)} d u \geq \int_{s}^{t} P(u) \frac{x(\tau(u))}{x(u)} d u,
$$


or

$$
\ln \frac{x(s)}{x(t)} \geq \int_{s}^{t} P(u) \frac{x(\tau(u))}{x(u)} d u \geq \int_{s}^{t} P(u) \exp \left(\int_{\tau(u)}^{u} G_{0}(\xi, \epsilon) d \xi\right) d u
$$

or

$$
x(s) \geq x(t) \exp \left(\int_{s}^{t} P(u) \exp \left(\int_{\tau(u)}^{u} G_{0}(\xi, \epsilon) d \xi\right) d u\right) .
$$

Setting $s=\tau(s)$ in $(2.13)$ we take

$$
x(\tau(s)) \geq x(t) \exp \left(\int_{\tau(s)}^{t} P(u) \exp \left(\int_{\tau(u)}^{u} G_{0}(\xi, \epsilon) d \xi\right) d u\right) .
$$

Combining (2.8) and (2.14) we obtain

$$
x(t)-x(\tau(t))+x(t) \int_{\tau(t)}^{t} P(s) \exp \left(\int_{\tau(s)}^{t} P(u) \exp \left(\int_{\tau(u)}^{u} G_{0}(\xi, \epsilon) d \xi\right) d u\right) d s \leq 0 .
$$

Multiplying the last inequality by $P(t)$, we find

$$
\begin{aligned}
P(t) x(t) & -P(t) x(\tau(t)) \\
& +P(t) x(t) \int_{\tau(t)}^{t} P(s) \exp \left(\int_{\tau(s)}^{t} P(u) \exp \left(\int_{\tau(u)}^{u} G_{0}(\xi, \epsilon) d \xi\right) d u\right) d s \leq 0,
\end{aligned}
$$

which, in view of (2.10), becomes

$x^{\prime}(t)+P(t) x(t)+P(t) x(t) \int_{\tau(t)}^{t} P(s) \exp \left(\int_{\tau(s)}^{t} P(u) \exp \left(\int_{\tau(u)}^{u} G_{0}(\xi, \epsilon) d \xi\right) d u\right) d s \leq 0$.

Hence, for sufficiently large $t$

$$
x^{\prime}(t)+P(t)\left[1+\int_{\tau(t)}^{t} P(s) \exp \left(\int_{\tau(s)}^{t} P(u) \exp \left(\int_{\tau(u)}^{u} G_{0}(\xi, \epsilon) d \xi\right) d u\right) d s\right] x(t) \leq 0,
$$

or

$$
x^{\prime}(t)+G_{1}(t, \epsilon) x(t) \leq 0
$$

where

$$
G_{1}(t, \epsilon)=P(t)\left[1+\int_{\tau(t)}^{t} P(s) \exp \left(\int_{\tau(s)}^{t} P(u) \exp \left(\int_{\tau(u)}^{u} G_{0}(\xi, \epsilon) d \xi\right) d u\right) d s\right] .
$$

It becomes apparent, now, that by repeating the above steps, we can build inequalities on $x^{\prime}(t)$ with progressively higher indices $G_{\ell}(t), \ell \in \mathbb{N}$. In general, for sufficiently large $\mathrm{t}$, the positive solution $x(t)$ satisfies the inequality

$$
x^{\prime}(t)+G_{\ell}(t, \epsilon) x(t) \leq 0, \quad(\ell \in \mathbb{N}),
$$


where

$G_{\ell}(t, \epsilon)=P(t)\left[1+\left[1+\int_{\tau(t)}^{t} P(s) \exp \left(\int_{\tau(s)}^{t} P(u) \exp \left(\int_{\tau(u)}^{u} G_{\ell-1}(\xi, \epsilon) d \xi\right) d u\right) d s\right]\right]$ and

$$
x(\tau(s)) \geq x(h(t)) \exp \left(\int_{\tau(s)}^{h(t)} P(u) \exp \left(\int_{\tau(u)}^{u} G_{\ell}(\xi, \epsilon) d \xi\right) d u\right) .
$$

Integrating $(E)$ from $h(t)$ to $t$, and using (2.16), we have

$0=x(t)-x(h(t))+\int_{h(t)}^{t} \sum_{i=1}^{m} p_{i}(s) x\left(\tau_{i}(s)\right) d s \geq x(t)-x(h(t))+\int_{h(t)}^{t} P(s) x(\tau(s)) d s$

or

$x(t)-x(h(t))+x(h(t)) \int_{h(t)}^{t} P(s) \exp \left(\int_{\tau(s)}^{h(t)} P(u) \exp \left(\int_{\tau(u)}^{u} G_{\ell}(\xi, \epsilon) d \xi\right) d u\right) d s \leq 0$.

The inequality is valid if we omit $x(t)>0$ in the left-hand side. Therefore

$$
x(h(t))\left[\int_{h(t)}^{t} P(s) \exp \left(\int_{\tau(s)}^{h(t)} P(u) \exp \left(\int_{\tau(u)}^{u} G_{\ell}(\xi, \epsilon) d \xi\right) d u\right) d s-1\right]<0,
$$

which means that

$$
\limsup _{t \rightarrow \infty} \int_{h(t)}^{t} P(s) \exp \left(\int_{\tau(s)}^{h(t)} P(u) \exp \left(\int_{\tau(u)}^{u} G_{\ell}(\xi, \epsilon) d \xi\right) d u\right) d s \leq 1 .
$$

Since $\epsilon$ may be taken arbitrarily small, this inequality contradicts (2.4).

The proof of the theorem is complete.

Theorem 5. Assume that $h(t)$ is defined by (1.6) and for some $\ell \in \mathbb{N}$

$$
\limsup _{t \rightarrow \infty} \int_{h(t)}^{t} P(s) \exp \left(\int_{\tau(s)}^{h(t)} P(u) \exp \left(\int_{\tau(u)}^{u} G_{\ell}(\xi) d \xi\right) d u\right) d s>1-D(\alpha)
$$

where $G_{\ell}$ is defined by (2.5). Then all solutions of $(E)$ are oscillatory.

Proof. Let $x$ be an eventually positive solution of $(E)$. Then, as in the proof of Theorem 1, (2.17) is satisfied, i.e.,

$x(t)-x(h(t))+x(h(t)) \int_{h(t)}^{t} P(s) \exp \left(\int_{\tau(s)}^{h(t)} P(u) \exp \left(\int_{\tau(u)}^{u} G_{\ell}(\xi, \epsilon) d \xi\right) d u\right) d s \leq 0$.

That is,

$$
\int_{h(t)}^{t} P(s) \exp \left(\int_{\tau(s)}^{h(t)} P(u) \exp \left(\int_{\tau(u)}^{u} G_{\ell}(\xi, \epsilon) d \xi\right) d u\right) d s \leq 1-\frac{x(t)}{x(h(t))},
$$


which gives

$$
\limsup _{t \rightarrow \infty} \int_{h(t)}^{t} P(s) \exp \left(\int_{\tau(s)}^{h(t)} P(u) \exp \left(\int_{\tau(u)}^{u} G_{\ell}(\xi, \epsilon) d \xi\right) d u\right) d s \leq 1-\liminf _{t \rightarrow \infty} \frac{x(t)}{x(h(t))} \text {. }
$$

By combining Lemmas 1 and 2, it becomes obvious that inequality (2.2) is fulfilled. So, (2.19) leads to

$$
\limsup _{t \rightarrow \infty} \int_{h(t)}^{t} P(s) \exp \left(\int_{\tau(s)}^{h(t)} P(u) \exp \left(\int_{\tau(u)}^{u} G_{\ell}(\xi, \epsilon) d \xi\right) d u\right) d s \leq 1-D(\alpha) .
$$

Since $\epsilon$ may be taken arbitrarily small, this inequality contradicts (2.18).

The proof of the theorem is complete.

Theorem 6. Assume that $h(t)$ is defined by (1.6) and for some $\ell \in \mathbb{N}$

$$
\limsup _{t \rightarrow \infty} \int_{h(t)}^{t} P(s) \exp \left(\int_{\tau(s)}^{t} P(u) \exp \left(\int_{\tau(u)}^{u} G_{\ell}(\xi) d \xi\right) d u\right) d s>\frac{1}{D(\alpha)}-1,
$$

where $G_{\ell}$ is defined by (2.5). Then all solutions of $(E)$ are oscillatory.

Proof. Assume, for the sake of contradiction, that there exists a nonoscillatory solution $x$ of $(E)$ and that $x$ is eventually positive. Then, as in the proof of Theorem 1 , for sufficiently large $t$ we have

$$
x(\tau(s)) \geq x(t) \exp \left(\int_{\tau(s)}^{t} P(u) \exp \left(\int_{\tau(u)}^{u} G_{\ell}(\xi, \epsilon) d \xi\right) d u\right) .
$$

Integrating $(E)$ from $h(t)$ to $t$ and in view of $(2.21)$, we have

$$
\begin{aligned}
0= & x(t)-x(h(t))+\int_{h(t)}^{t} \sum_{i=1}^{m} p_{i}(s) x\left(\tau_{i}(s)\right) d s \geq x(t)-x(h(t)) \\
& +\int_{h(t)}^{t} P(s) x(\tau(s)) d s \\
\geq & x(t)-x(h(t)) \\
& +x(h(t)) \int_{h(t)}^{t} P(s) \frac{x(t)}{x(h(t))} \exp \left(\int_{\tau(s)}^{t} P(u) \exp \left(\int_{\tau(u)}^{u} G_{\ell}(\xi, \epsilon) d \xi\right) d u\right) d s .
\end{aligned}
$$

That is, for all sufficiently large $t$ it holds

$$
\int_{h(t)}^{t} P(s) \exp \left(\int_{\tau(s)}^{t} P(u) \exp \left(\int_{\tau(u)}^{u} G_{\ell}(\xi, \epsilon) d \xi\right) d u\right) d s \leq \frac{x(h(t))}{x(t)}-1
$$

and therefore

$$
\limsup _{t \rightarrow \infty} \int_{h(t)}^{t} P(s) \exp \left(\int_{\tau(s)}^{t} P(u) \exp \left(\int_{\tau(u)}^{u} G_{\ell}(\xi, \epsilon) d \xi\right) d u\right) d s \leq \limsup _{t \rightarrow \infty} \frac{x(h(t))}{x(t)}-1 .
$$


By combining Lemmas 1 and 2, it becomes obvious that inequality (2.2) is fulfilled. So, (2.22) leads to

$$
\limsup _{t \rightarrow \infty} \int_{h(t)}^{t} P(s) \exp \left(\int_{\tau(s)}^{t} P(u) \exp \left(\int_{\tau(u)}^{u} G_{\ell}(\xi, \epsilon) d \xi\right) d u\right) d s \leq \frac{1}{D(\alpha)}-1 .
$$

Since $\epsilon$ may be taken arbitrarily small, this inequality contradicts (2.20).

The proof of the theorem is complete.

Theorem 7. Assume that $h(t)$ is defined by (1.6) and for some $\ell \in \mathbb{N}$

$$
\limsup _{t \rightarrow \infty} \int_{h(t)}^{t} P(s) \exp \left(\int_{\tau(s)}^{h(s)} P(u) \exp \left(\int_{\tau(u)}^{u} G_{\ell}(\xi) d \xi\right) d u\right) d s>\frac{1+\ln \lambda_{0}}{\lambda_{0}}-D(\alpha),
$$

where $G_{\ell}$ is defined by (2.5) and $\lambda_{0}$ is the smaller root of the transcendental equation $\lambda=e^{\alpha \lambda}$. Then all solutions of $(E)$ are oscillatory.

Proof. Assume, for the sake of contradiction, that there exists a nonoscillatory solution $x$ of $(E)$ and that $x$ is eventually positive. Then, as in the previous theorems, (2.21) holds.

Observe that (2.3) implies that for each $\epsilon>0$ there exists a $t_{\epsilon}$ such that

$$
\lambda_{0}-\epsilon<\frac{x(h(t))}{x(t)} \text { for all } t \geq t_{\epsilon} .
$$

Noting that by nondecreasing nature of the function $\frac{x(h(t))}{x(s)}$ in $s$, it holds

$$
1=\frac{x(h(t))}{x(h(t))} \leq \frac{x(h(t))}{x(s)} \leq \frac{x(h(t))}{x(t)}, \quad t_{\varepsilon} \leq h(t) \leq s \leq t
$$

in particular for $\epsilon \in\left(0, \lambda_{0}-1\right)$, by continuity we see that there exists a $t^{*} \in(h(t), t]$ such that

$$
1<\lambda_{0}-\epsilon=\frac{x(h(t))}{x\left(t^{*}\right)} .
$$

By (2.21), it is obvious that

$$
x(\tau(s)) \geq x(h(s)) \exp \left(\int_{\tau(s)}^{h(s)} P(u) \exp \left(\int_{\tau(u)}^{u} G_{\ell}(\xi, \epsilon) d \xi\right) d u\right) .
$$

Integrating ( $E$ ) from $t^{*}$ to $t$ and using (2.26) we have

$$
\begin{aligned}
0 & =x(t)-x\left(t^{*}\right)+\int_{t^{*}}^{t} \sum_{i=1}^{m} p_{i}(s) x\left(\tau_{i}(s)\right) d s \\
& \geq x(t)-x\left(t^{*}\right)+\int_{t^{*}}^{t}\left(\sum_{i=1}^{m} p_{i}(s)\right) x(\tau(s)) d s
\end{aligned}
$$




$$
\begin{aligned}
& =x(t)-x\left(t^{*}\right)+\int_{t^{*}}^{t} P(s) x(\tau(s)) d s \\
& \geq x(t)-x\left(t^{*}\right)+x(h(t)) \int_{t^{*}}^{t} P(s) \exp \left(\int_{\tau(s)}^{h(s)} P(u) \exp \left(\int_{\tau(u)}^{u} G_{\ell}(\xi, \epsilon) d \xi\right) d u\right) d s
\end{aligned}
$$

or

$$
\int_{t^{*}}^{t} P(s) \exp \left(\int_{\tau(s)}^{h(s)} P(u) \exp \left(\int_{\tau(u)}^{u} G_{\ell}(\xi, \epsilon) d \xi\right) d u\right) d s \leq \frac{x\left(t^{*}\right)}{x(h(t))}-\frac{x(t)}{x(h(t))} .
$$

In view of (2.25) and Lemma 2 , for the $\epsilon$ considered, there exists $t_{\epsilon}^{\prime} \geq t_{\varepsilon}$ such that

$$
\int_{t^{*}}^{t} P(s) \exp \left(\int_{\tau(s)}^{h(s)} P(u) \exp \left(\int_{\tau(u)}^{u} G_{\ell}(\xi, \epsilon) d \xi\right) d u\right) d s<\frac{1}{\lambda_{0}-\epsilon}-D(\alpha)+\epsilon,
$$

for $t \geq t_{\epsilon}^{\prime}$.

Dividing $(E)$ by $x(t)$ and integrating from $h(t)$ to $t^{*}$ we find

$$
\begin{aligned}
& -\int_{h(t)}^{t^{*}} \frac{x^{\prime}(s)}{x(s)} d s \\
& =\int_{h(t)}^{t^{*}} \sum_{i=1}^{m} p_{i}(s) \frac{x\left(\tau_{i}(s)\right)}{x(s)} d s \geq \int_{h(t)}^{t^{*}}\left(\sum_{i=1}^{m} p_{i}(s)\right) \frac{x(\tau(s))}{x(s)} d s \\
& \geq \int_{h(t)}^{t^{*}} P(s) \frac{x(h(s))}{x(s)} \exp \left(\int_{\tau(s)}^{h(s)} P(u) \exp \left(\int_{\tau(u)}^{u} G_{\ell}(\xi, \epsilon) d \xi\right) d u\right) d s .
\end{aligned}
$$

By $(2.25)$, for $s \geq h(t) \geq t_{\epsilon}^{\prime}$, we have $\frac{x(h(s))}{x(s)}>\lambda_{0}-\epsilon$, so from $(2.28)$ we get

$$
\left(\lambda_{0}-\epsilon\right) \int_{h(t)}^{t^{*}} P(s) \exp \left(\int_{\tau(s)}^{h(s)} P(u) \exp \left(\int_{\tau(u)}^{u} G_{\ell}(\xi, \epsilon) d \xi\right) d u\right) d s<-\int_{h(t)}^{t^{*}} \frac{x^{\prime}(s)}{x(s)} d s .
$$

Hence, for all sufficiently large $t$ we have

$$
\begin{aligned}
\int_{h(t)}^{t^{*}} P(s) \exp \left(\int_{\tau(s)}^{h(s)}\right. & \left.P(u) \exp \left(\int_{\tau(u)}^{u} G_{\ell}(\xi, \epsilon) d \xi\right) d u\right) d s \\
& <-\frac{1}{\lambda_{0}-\epsilon} \int_{h(t)}^{t^{*}} \frac{x^{\prime}(s)}{x(s)} d s=\frac{1}{\lambda_{0}-\epsilon} \ln \frac{x(h(t))}{x\left(t^{*}\right)}=\frac{\ln \left(\lambda_{0}-\epsilon\right)}{\lambda_{0}-\epsilon},
\end{aligned}
$$

i.e.,

$$
\int_{h(t)}^{t^{*}} P(s) \exp \left(\int_{\tau(s)}^{h(s)} P(u) \exp \left(\int_{\tau(u)}^{u} G_{\ell}(\xi, \epsilon) d \xi\right) d u\right) d s<\frac{\ln \left(\lambda_{0}-\epsilon\right)}{\lambda_{0}-\epsilon} .
$$

Adding (2.27) and (2.29), and then taking the limit as $t \rightarrow \infty$, we have 


$$
\begin{aligned}
\limsup _{t \rightarrow \infty} \int_{h(t)}^{t} P(s) \exp \left(\int_{\tau(s)}^{h(s)} P(u)\right. & \left.\exp \left(\int_{\tau(u)}^{u} G_{\ell}(\xi, \epsilon) d \xi\right) d u\right) d s \\
\leq & \frac{1+\ln \left(\lambda_{0}-\epsilon\right)}{\lambda_{0}-\epsilon}-\frac{1-\alpha-\sqrt{1-2 \alpha-\alpha^{2}}}{2}+\epsilon .
\end{aligned}
$$

Since $\epsilon$ may be taken arbitrarily small, this inequality contradicts (2.23).

The proof of the theorem is complete.

Theorem 8. Assume that $h(t)$ is defined by (1.6) and for some $\ell \in \mathbb{N}$

$$
\liminf _{t \rightarrow \infty} \int_{h(t)}^{t} P(s) \exp \left(\int_{\tau(s)}^{h(s)} P(u) \exp \left(\int_{\tau(u)}^{u} G_{\ell}(\xi) d \xi\right) d u\right) d s>\frac{1}{e},
$$

where $G_{\ell}$ is defined by (2.5). Then all solutions of $(E)$ are oscillatory.

Proof. Assume, for the sake of contradiction, that there exists a nonoscillatory solution $x(t)$ of $(E)$. Since $-x(t)$ is also a solution of $(E)$, we can confine our discussion only to the case where the solution $x(t)$ is eventually positive. Then there exists $t_{1}>t_{0}$ such that $x(t), x\left(\tau_{i}(t)\right)>0,1 \leq i \leq m$ for all $t \geq t_{1}$. Thus, from $(E)$ we have

$$
x^{\prime}(t)=-\sum_{i=1}^{m} p_{i}(t) x\left(\tau_{i}(t)\right) \leq 0, \quad \text { for all } t \geq t_{1},
$$

which means that $x(t)$ is an eventually nonincreasing function of positive numbers. Furthermore, as in previous theorem, (2.26) is satisfied.

Dividing $(E)$ by $x(t)$ and integrating from $h(t)$ to $t$, for some $t_{2} \geq t_{1}$, we have

$$
\begin{aligned}
\ln \left(\frac{x(h(t))}{x(t)}\right) & =\int_{h(t)}^{t} \sum_{i=1}^{m} p_{i}(s) \frac{x\left(\tau_{i}(s)\right)}{x(s)} d s \\
& \geq \int_{h(t)}^{t}\left(\sum_{i=1}^{m} p_{i}(s)\right) \frac{x(\tau(s))}{x(s)} d s=\int_{h(t)}^{t} P(s) \frac{x(\tau(s))}{x(s)} d s .
\end{aligned}
$$

Combining the inequalities (2.26) and (2.31) we obtain

$$
\ln \left(\frac{x(h(t))}{x(t)}\right) \geq \int_{h(t)}^{t} P(s) \frac{x(h(s))}{x(s)} \exp \left(\int_{\tau(s)}^{h(s)} P(u) \exp \left(\int_{\tau(u)}^{u} G_{\ell}(\xi, \epsilon) d \xi\right) d u\right) d s
$$

From (2.30), it follows that there exists a constant $c>0$ such that for a sufficiently large $t$ holds

$$
\int_{h(t)}^{t} P(s) \exp \left(\int_{\tau(s)}^{h(s)} P(u) \exp \left(\int_{\tau(u)}^{u} G_{\ell}(\xi) d \xi\right) d u\right) d s \geq c>\frac{1}{e} .
$$

Choose $c^{\prime}$ such that $c>c^{\prime}>1 / e$. For every $\epsilon>0$ such that $c-\epsilon>c^{\prime}$ we have

$$
\int_{h(t)}^{t} P(s) \exp \left(\int_{\tau(s)}^{h(s)} P(u) \exp \left(\int_{\tau(u)}^{u} G_{\ell}(\xi, \epsilon) d \xi\right) d u\right) d s \geq c-\epsilon>c^{\prime}>\frac{1}{e}
$$


Hence

$$
\ln \left(\frac{x(h(t))}{x(t)}\right) \geq c^{\prime}, \quad t \geq t_{3}
$$

Thus

$$
\frac{x(h(t))}{x(t)} \geq e^{c^{\prime}} \geq e c^{\prime}>1
$$

which implies for some $t \geq t_{4} \geq t_{3}$

$$
x(h(t)) \geq\left(e c^{\prime}\right) x(t)
$$

Repeating the above procedure, it follows by induction that for any positive integer $k$,

$$
\frac{x(h(t))}{x(t)} \geq\left(e c^{\prime}\right)^{k}, \quad \text { for sufficiently large } t .
$$

Since $e c^{\prime}>1$, there is $k \in \mathbb{N}$ satisfying $k>2\left(\ln (2)-\ln \left(c^{\prime}\right)\right) /\left(1+\ln \left(c^{\prime}\right)\right)$ such that for $t$ sufficiently large

$$
\frac{x(h(t))}{x(t)} \geq\left(e c^{\prime}\right)^{k}>\left(\frac{2}{c^{\prime}}\right)^{2} .
$$

Taking the integral on $[h(t), t]$, which is not less than $c^{\prime}$, we split the interval into two parts where integrals are not less than $c^{\prime} / 2$, let $t_{m} \in(h(t), t)$ be the splitting point:

$$
\begin{aligned}
& \int_{h(t)}^{t_{m}} P(s) \exp \left(\int_{\tau(s)}^{h(s)} P(u) \exp \left(\int_{\tau(u)}^{u} G_{\ell}(\xi, \epsilon) d \xi\right) d u\right) d s \geq \frac{c^{\prime}}{2}, \\
& \int_{t_{m}}^{t} P(s) \exp \left(\int_{\tau(s)}^{h(s)} P(u) \exp \left(\int_{\tau(u)}^{u} G_{\ell}(\xi, \epsilon) d \xi\right) d u\right) d s \geq \frac{c^{\prime}}{2} .
\end{aligned}
$$

Integrating $(E)$ from $t_{m}$ to $t$, gives

$$
x(t)-x\left(t_{m}\right)+\int_{t_{m}}^{t} \sum_{i=1}^{m} p_{i}(s) x\left(\tau_{i}(s)\right)=0,
$$

or

$$
x(t)-x\left(t_{m}\right)+\int_{t_{m}}^{t}\left(\sum_{i=1}^{m} p_{i}(s)\right) x(\tau(s)) \leq 0 .
$$

Thus

$$
x(t)-x\left(t_{m}\right)+\int_{t_{m}}^{t} P(s) x(\tau(s)) \leq 0,
$$

or

$$
x(t)-x\left(t_{m}\right)+x(h(t)) \int_{t_{m}}^{t} P(s) \exp \left(\int_{\tau(s)}^{h(s)} P(u) \exp \left(\int_{\tau(u)}^{u} G_{\ell}(\xi, \epsilon) d \xi\right) d u\right) d s \leq 0 .
$$

The strict inequality is valid if we omit $x(t)>0$ in the left-hand side:

$$
-x\left(t_{m}\right)+x(h(t)) \int_{t_{m}}^{t} P(s) \exp \left(\int_{\tau(s)}^{h(s)} P(u) \exp \left(\int_{\tau(u)}^{u} G_{\ell}(\xi, \epsilon) d \xi\right) d u\right) d s<0 .
$$


Together with the second inequality in (2.34), implies

$$
x\left(t_{m}\right)>\frac{c^{\prime}}{2} x(h(t))
$$

Similarly, integration of $(E)$ from $h(t)$ to $t_{m}$ with a later application of $(2.26)$ leads to

$$
x\left(t_{m}\right)-x(h(t))+x\left(h\left(t_{m}\right)\right) \int_{h(t)}^{t_{m}} P(s) \exp \left(\int_{\tau(s)}^{h(s)} P(u) \exp \left(\int_{\tau(u)}^{u} G_{\ell}(\xi, \epsilon) d \xi\right) d u\right) d s \leq 0 .
$$

The strict inequality is valid if we omit $x\left(t_{m}\right)>0$ in the left-hand side:

$$
-x(h(t))+x\left(h\left(t_{m}\right)\right) \int_{h(t)}^{t_{m}} P(s) \exp \left(\int_{\tau(s)}^{h(s)} P(u) \exp \left(\int_{\tau(u)}^{u} G_{\ell}(\xi, \epsilon) d \xi\right) d u\right) d s<0 .
$$

Together with the first inequality in (2.34) implies

$$
x(h(t))>\frac{c^{\prime}}{2} x\left(h\left(t_{m}\right)\right) .
$$

Combining the inequalities (2.35) and (2.36) we obtain

$$
x\left(h\left(t_{m}\right)\right)<\frac{2}{c^{\prime}} x(h(t))<\left(\frac{2}{c^{\prime}}\right)^{2} x\left(t_{m}\right),
$$

which contradicts (2.33).

The proof of the theorem is complete.

\subsection{ADVANCED DIFFERENTIAL EQUATIONS}

Similar oscillation conditions for the (dual) advanced differential equation $\left(E^{\prime}\right)$ can be derived easily. The proofs are omitted, since they are quite similar to the delay equation.

Theorem 9. Assume that $\rho(t)$ is defined by (1.20) and for some $\ell \in \mathbb{N}$

$$
\limsup _{t \rightarrow \infty} \int_{t}^{\rho(t)} Q(s) \exp \left(\int_{\rho(t)}^{\sigma(s)} Q(u) \exp \left(\int_{u}^{\sigma(u)} R_{\ell}(\xi) d \xi\right) d u\right) d s>1,
$$

where

$$
R_{\ell}(t)=Q(t)\left[1+\int_{t}^{\rho(t)} Q(s) \exp \left(\int_{t}^{\sigma(s)} Q(u) \exp \left(\int_{u}^{\sigma(u)} R_{\ell-1}(\xi) d \xi\right) d u\right) d s\right]
$$

with $R_{0}(t)=Q(t)\left[1+\int_{t}^{\rho(t)} Q(s) \exp \left(\lambda_{0} \int_{t}^{\sigma(s)} Q(u) d u\right) d s\right], Q(t)=\sum_{i=1}^{m} q_{i}(t)$ and $\lambda_{0}$ is the smaller root of the transcendental equation $\lambda=e^{\beta \lambda}$. Then all solutions of $\left(E^{\prime}\right)$ are oscillatory. 
Theorem 10. Assume that $\rho(t)$ is defined by (1.20) and for some $\ell \in \mathbb{N}$

$$
\limsup _{t \rightarrow \infty} \int_{t}^{\rho(t)} Q(s) \exp \left(\int_{\rho(t)}^{\sigma(s)} Q(u) \exp \left(\int_{u}^{\sigma(u)} R_{\ell}(\xi) d \xi\right) d u\right) d s>1-D(\beta),
$$

where $R_{\ell}$ is defined by (2.38) Then all solutions of $\left(E^{\prime}\right)$ are oscillatory.

Theorem 11. Assume that $\rho(t)$ is defined by (1.20) and for some $\ell \in \mathbb{N}$

$$
\limsup _{t \rightarrow \infty} \int_{t}^{\rho(t)} Q(s) \exp \left(\int_{t}^{\sigma(s)} Q(u) \exp \left(\int_{u}^{\sigma(u)} R_{\ell}(\xi) d \xi\right) d u\right) d s>\frac{1}{D(\beta)}-1,
$$

where $R_{\ell}$ is defined by (2.38) Then all solutions of $\left(E^{\prime}\right)$ are oscillatory.

Theorem 12. Assume that $\rho(t)$ is defined by (1.20) and for some $\ell \in \mathbb{N}$

$\limsup _{t \rightarrow \infty} \int_{t}^{\rho(t)} Q(s) \exp \left(\int_{\rho(s)}^{\sigma(s)} Q(u) \exp \left(\int_{u}^{\sigma(u)} R_{\ell}(\xi) d \xi\right) d u\right) d s>\frac{1+\ln \lambda_{0}}{\lambda_{0}}-D(\beta)$,

where $R_{\ell}$ is defined by (2.38) and $\lambda_{0}$ is the smaller root of the transcendental equation $\lambda=e^{\beta \lambda}$. Then all solutions of $\left(E^{\prime}\right)$ are oscillatory.

Theorem 13. Assume that $\rho(t)$ is defined by (1.20) and for some $\ell \in \mathbb{N}$

$$
\liminf _{t \rightarrow \infty} \int_{t}^{\rho(t)} Q(s) \exp \left(\int_{\rho(s)}^{\sigma(s)} Q(u) \exp \left(\int_{u}^{\sigma(u)} R_{\ell}(\xi) d \xi\right) d u\right) d s>\frac{1}{e},
$$

where $R_{\ell}$ is defined by (2.38) Then all solutions of $\left(E^{\prime}\right)$ are oscillatory.

\subsection{DIFFERENTIAL INEQUALITIES}

A slight modification in the proofs of Theorems 1 [6]-5 [10] leads to the following results about differential inequalities.

Theorem 14. Assume that all the conditions of Theorem 1 [6] or 2 [7] or 3 [8] or 4 [9] or 5 [10] hold. Then

(i) the delay [advanced] differential inequality

$$
x^{\prime}(t)+\sum_{i=1}^{m} p_{i}(t) x\left(\tau_{i}(t)\right) \leq 0 \quad\left[x^{\prime}(t)-\sum_{i=1}^{m} q_{i}(t) x\left(\sigma_{i}(t)\right) \geq 0\right], \forall t \geq t_{0},
$$

has no eventually positive solutions;

(ii) the delay [advanced] differential inequality

$$
x^{\prime}(t)+\sum_{i=1}^{m} p_{i}(t) x\left(\tau_{i}(t)\right) \geq 0 \quad\left[x^{\prime}(t)-\sum_{i=1}^{m} q_{i}(t) x\left(\sigma_{i}(t)\right) \leq 0\right], \forall t \geq t_{0},
$$

has no eventually negative solutions. 


\section{EXAMPLES}

In this section, examples illustrate cases when the results of the present paper imply oscillation while previously known results fail. The calculations were made by the use of MATLAB software.

Example 15. Consider the DDE

$$
x^{\prime}(t)+\frac{177}{2000} x\left(\tau_{1}(t)\right)+\frac{59}{2000} x\left(\tau_{2}(t)\right)=0, \quad t \geq 0,
$$

with (see Fig. 1, (a))

$$
\tau_{1}(t)=\left\{\begin{array}{ll}
-3 t+24 k-2, & \text { if } t \in[6 k, 6 k+1] \\
5 t-24 k-10, & \text { if } t \in[6 k+1,6 k+2] \\
6 k, & \text { if } t \in[6 k+2,6 k+3] \\
-2 t+18 k+6, & \text { if } t \in[6 k+3,6 k+4] \\
6 t-30 k-26, & \text { if } t \in[6 k+4,6 k+5] \\
6 k+4, & \text { if } t \in[6 k+5,6 k+6]
\end{array} \text { and } \tau_{2}(t)=\tau_{1}(t)-0.5\right.
$$

where $k \in \mathbb{N}_{0}$ and $\mathbb{N}_{0}$ is the set of non-negative integers.
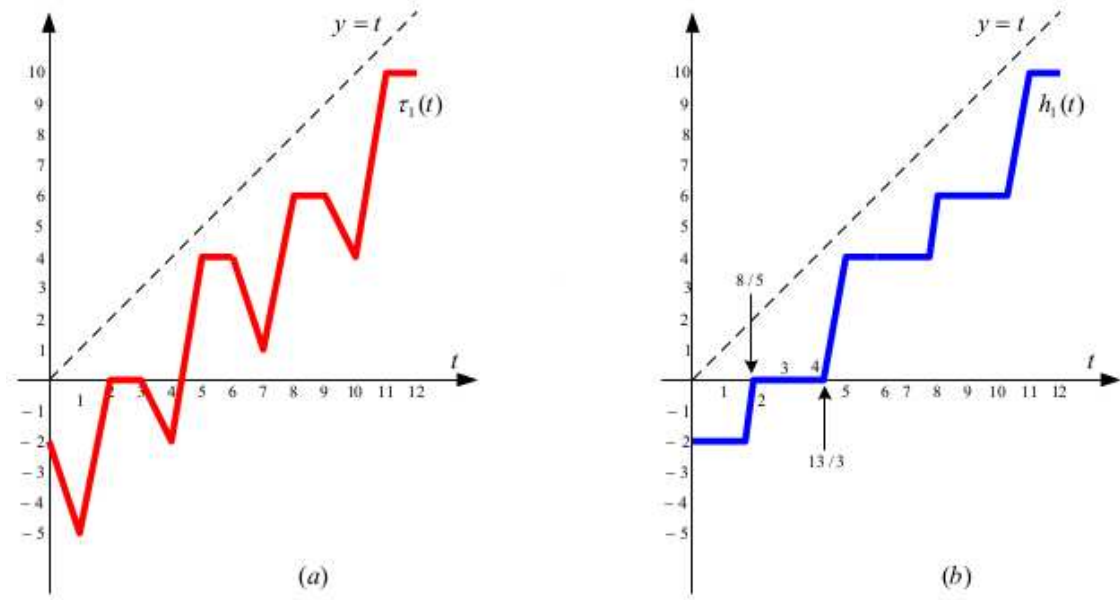

Figure 1: The graphs of $\tau_{1}(t)$ and $h_{1}(t)$ 
By (1.6), we see (Fig. 1, (b)) that

$$
h_{1}(t)=\left\{\begin{array}{ll}
6 k-2, & \text { if } t \in[6 k, 6 k+8 / 5] \\
5 t-24 k-10, & \text { if } t \in[6 k+8 / 5,6 k+2] \\
6 k, & \text { if } t \in[6 k+2,6 k+13 / 3] \\
6 t-30 k-26, & \text { if } t \in[6 k+13 / 3,6 k+5] \\
6 k+4, & \text { if } t \in[6 k+5,6 k+6]
\end{array} \quad \text { and } \quad h_{2}(t)=h_{1}(t)-0.5\right.
$$

and consequently

$$
h(t)=\max _{1 \leq i \leq 2}\left\{h_{i}(t)\right\}=h_{1}(t) \quad \text { and } \quad \tau(t)=\max _{1 \leq i \leq 2}\left\{\tau_{i}(t)\right\}=\tau_{1}(t)
$$

Observe that the function $F_{\ell}:[0, \infty) \rightarrow \mathbb{R}_{+}$defined as

$$
F_{\ell}(t)=\int_{h(t)}^{t} P(s) \exp \left(\int_{\tau(s)}^{h(t)} P(u) \exp \left(\int_{\tau(u)}^{u} G_{\ell}(\xi) d \xi\right) d u\right) d s
$$

attains its maximum at $t=6 k+13 / 3, k \in \mathbb{N}_{0}$, for every $\ell \geq 1$. Specifically, by using algorithms on MATLAB software and taking into account the fact that $P(t)=$ $\sum_{i=1}^{2} p_{i}(t)=0.118$, we obtain

$$
F_{1}(t=6 k+13 / 3)=\int_{6 k}^{6 k+13 / 3} P(s) \exp \left(\int_{\tau(s)}^{6 k} P(u) \exp \left(\int_{\tau(u)}^{u} G_{1}(\xi) d \xi\right) d u\right) d s
$$

Thus

$$
\limsup _{t \rightarrow \infty} F_{1}(t) \simeq 1.0461>1,
$$

that is, condition (2.4) of Theorem 1 is satisfied for $\ell=1$, and therefore all solutions of (3.1) oscillate.

Observe, however, that

$$
\begin{aligned}
& \limsup _{t \rightarrow \infty} \int_{h(t)}^{t} \sum_{i=1}^{2} p_{i}(s) d s=\limsup _{k \rightarrow \infty} \int_{6 k}^{6 k+13 / 3} \sum_{i=1}^{2} p_{i}(s) d s=0.5113<1, \\
& \alpha=\liminf _{t \rightarrow \infty} \int_{\tau(t)}^{t} \sum_{i=1}^{2} p_{i}(s) d s=\liminf _{k \rightarrow \infty} \int_{6 k+4}^{6 k+5} \sum_{i=1}^{2} p_{i}(s) d s=0.118<\frac{1}{e}, \\
& \quad \liminf _{t \rightarrow \infty} \sum_{i=1}^{2} p_{i}(t)\left(t-\tau_{i}(t)\right) \\
& =\liminf _{t \rightarrow \infty}\left[\frac{177}{2000}\left(t-\tau_{1}(t)\right)+\frac{59}{2000}\left(t-\left(\tau_{1}(t)-0.5\right)\right)\right] \\
& =\liminf _{t \rightarrow \infty}\left[0.118\left(t-\tau_{1}(t)\right)+0.01475\right]=\liminf _{t \rightarrow \infty}\left[0.118\left(t-\tau_{1}(t)\right)\right]+0.01475
\end{aligned}
$$




$$
=0.118 \cdot \liminf _{t \rightarrow \infty}\left(t-\tau_{1}(t)\right)+0.01475=0.118 \cdot 1+0.01475=0.13275<\frac{1}{e} .
$$

Also, observe that the function $\Phi_{r}:[0, \infty) \rightarrow \mathbb{R}_{+}$defined as

$$
\Phi_{r}(t)=\int_{h(t)}^{t} \sum_{i=1}^{m} p_{i}(\zeta) a_{r}\left(h(t), \tau_{i}(\zeta)\right) d \zeta
$$

attains its maximum at $t=6 k+13 / 3$ and its minimum at $t=6 k+5, k \in \mathbb{N}_{0}$, for every $r \in \mathbb{N}$. Specifically,

$$
\begin{aligned}
\Phi_{1}(t= & 6 k+13 / 3)=\int_{6 k}^{6 k+13 / 3}\left[p_{1}(\zeta) a_{1}\left(6 k, \tau_{1}(\zeta)\right)+p_{2}(\zeta) a_{1}\left(6 k, \tau_{2}(\zeta)\right)\right] d \zeta \\
= & \int_{6 k}^{6 k+1}\left[p_{1}(\zeta) a_{1}\left(6 k, \tau_{1}(\zeta)\right)+p_{2}(\zeta) a_{1}\left(6 k, \tau_{2}(\zeta)\right)\right] d \zeta \\
& +\int_{6 k+1}^{6 k+2}\left[p_{1}(\zeta) a_{1}\left(6 k, \tau_{1}(\zeta)\right)+p_{2}(\zeta) a_{1}\left(6 k, \tau_{2}(\zeta)\right)\right] d \zeta \\
& +\int_{6 k+2}^{6 k+3}\left[p_{1}(\zeta) a_{1}\left(6 k, \tau_{1}(\zeta)\right)+p_{2}(\zeta) a_{1}\left(6 k, \tau_{2}(\zeta)\right)\right] d \zeta \\
& +\int_{6 k+3}^{6 k+4}\left[p_{1}(\zeta) a_{1}\left(6 k, \tau_{1}(\zeta)\right)+p_{2}(\zeta) a_{1}\left(6 k, \tau_{2}(\zeta)\right)\right] d \zeta \\
& +\int_{6 k+4}^{6 k+13 / 3}\left[p_{1}(\zeta) a_{1}\left(6 k, \tau_{1}(\zeta)\right)+p_{2}(\zeta) a_{1}\left(6 k, \tau_{2}(\zeta)\right)\right] d \zeta \\
\simeq & 0.6452
\end{aligned}
$$

and

$$
\begin{aligned}
\Phi_{1}(t & =6 k+5)=\int_{6 k+4}^{6 k+5}\left[p_{1}(\zeta) a_{1}\left(6 k+4, \tau_{1}(\zeta)\right)+p_{2}(\zeta) a_{1}\left(6 k+4, \tau_{2}(\zeta)\right)\right] d \zeta \\
& \simeq 0.1743 .
\end{aligned}
$$

Thus

$$
\begin{gathered}
\lim \sup _{t \rightarrow \infty} \Phi_{1}(t) \simeq 0.6452<1 \\
\liminf \inf _{t \rightarrow \infty} G_{1}(t) \simeq 0.1743<1 / e
\end{gathered}
$$

and

$$
0.6452<1-D(\alpha) \simeq 0.9920
$$

Also, it is obvious that

$$
\begin{aligned}
\limsup _{t \rightarrow \infty} \int_{h(t)}^{t} \sum_{i=1}^{2} p_{i}(\zeta) a_{r}\left(h(\zeta), \tau_{i}(\zeta)\right) d \zeta \leq \limsup _{t \rightarrow \infty} & \Phi_{1}(t) \simeq 0.6452 \\
& <\frac{1+\ln \lambda_{0}}{\lambda_{0}}-D(\alpha) \simeq 0.9837
\end{aligned}
$$

where $\lambda_{0}=1.14461$ is the smaller root of $e^{0.118 \lambda}=\lambda$. 
Finally, by using algorithms on MATLAB software, we obtain

$$
\begin{gathered}
\limsup _{t \rightarrow \infty} \int_{h(t)}^{t} \bar{P}(s) \exp \left(\int_{\tau(s)}^{h(t)} \bar{P}_{j}(u) d u\right) d s \simeq 0.7961<1, \\
0.7961<1-D(\alpha) \simeq 0.9920,
\end{gathered}
$$

$\limsup _{t \rightarrow \infty} \int_{h(t)}^{t} \bar{P}(s) \exp \left(\int_{\tau(s)}^{h(s)} \bar{P}_{j}(u) d u\right) d s \simeq 0.6384<\frac{1+\ln \lambda_{0}}{\lambda_{0}}-D(\alpha) \simeq 0.9837$,

where $\lambda_{0}=1.29586$ is the smaller root of $e^{\lambda \beta}=\lambda$,

$$
\limsup _{t \rightarrow \infty} \int_{h(t)}^{t} \bar{P}(s) \exp \left(\int_{\tau(s)}^{t} \bar{P}_{j}(u) d u\right) d s \simeq 2.5211<\frac{1}{D(\alpha)} \simeq 125.5436
$$

and

$$
\liminf _{t \rightarrow \infty} \int_{h(t)}^{t} \bar{P}(s) \exp \left(\int_{\tau(s)}^{h(s)} \bar{P}_{j}(u) d u\right) d s \simeq 0.1259<\frac{1}{e}
$$

That is, none of the conditions (1.3), (1.4), (1.6), (1.8) (for $r=1),(1.9)$ (for $r=1$ ), (1.10) (for $r=1$ ), (1.11) (for $r=1$ ), (1.12) (for $j=1$ ), (1.13) (for $j=1$ ), (1.14) (for $j=1$ ), (1.15) (for $j=1$ ) and (1.16) (for $j=1$ ) is satisfied.

Notation. It is worth noting that the improvement of condition (2.4) to the corresponding condition (1.3) is significant, approximately $104.6 \%$, if we compare the values on the left-side of these conditions. Also, the improvement compared to conditions (1.8) and (1.12) is very satisfactory, around $62.14 \%$ and $31.4 \%$, respectively. In addition, observe that conditions (1.8)-(1.9) and (1.10)-(1.16) do not lead to oscillation for first iteration. On the contrary, condition (2.4) is satisfied from the first iteration. This means that our condition is better and much faster than (1.8)-(1.9) and $(1.10)-(1.16)$.

Example 16. Consider the ADE

$$
x^{\prime}(t)-\frac{3}{20} x\left(\sigma_{1}(t)\right)-\frac{1}{20} x\left(\sigma_{2}(t)\right)=0, \quad t \geq 0,
$$

with (see Fig. 2, (a))

$$
\sigma_{1}(t)=\left\{\begin{array}{ll}
4 t-21 k+1, & \text { if } t \in[7 k, 7 k+1] \\
-t+14 k+6, & \text { if } t \in[7 k+1,7 k+2] \\
7 k+4, & \text { if } t \in[7 k+2,7 k+3] \\
3 t-14 k-5, & \text { if } t \in[7 k+3,7 k+4] \\
-t+14 k+11, & \text { if } t \in[7 k+4,7 k+5] \\
2 t-7 k-4, & \text { if } t \in[7 k+5,7 k+6] \\
7 k+8, & \text { if } t \in[7 k+6,7 k+7]
\end{array} \text { and } \sigma_{2}(t)=\sigma_{1}(t)+0.5\right.
$$




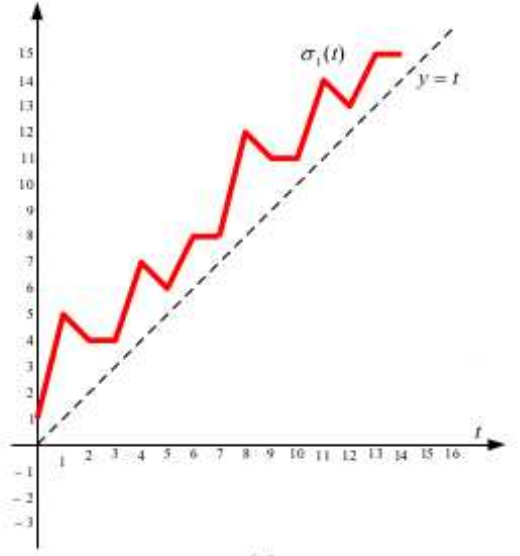

(a)

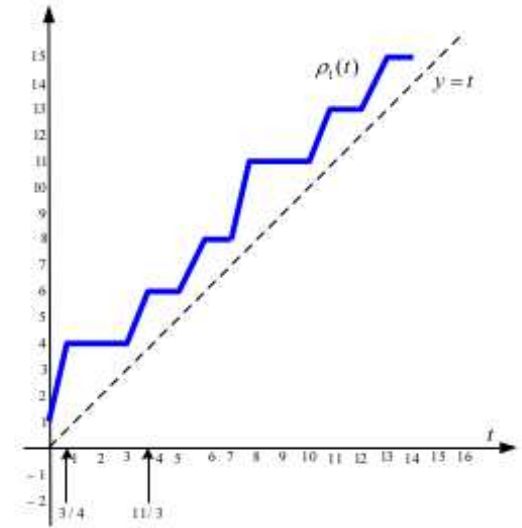

(b)

Figure 2: The graphs of $\sigma_{1}(t)$ and $\rho_{1}(t)$

where $k \in \mathbb{N}_{0}$ and $\mathbb{N}_{0}$ is the set of non-negative integers.

By (1.20), we see (Fig. 2, (b)) that

$$
\rho_{1}(t)=\left\{\begin{array}{ll}
4 t-21 k+1, & \text { if } t \in[7 k, 7 k+3 / 4] \\
7 k+4, & \text { if } t \in[7 k+3 / 4,7 k+3] \\
3 t-14 k-5, & \text { if } t \in[7 k+3,7 k+11 / 3] \\
7 k+6, & \text { if } t \in[7 k+11 / 3,7 k+5] \\
2 t-7 k-4, & \text { if } t \in[7 k+5,7 k+6] \\
7 k+8, & \text { if } t \in[7 k+6,7 k+7]
\end{array} \text { and } \quad \rho_{2}(t)=\rho_{1}(t)+0.5\right.
$$

and consequently

$$
\rho(t)=\min _{1 \leq i \leq 2}\left\{\rho_{i}(t)\right\}=\rho_{1}(t) \quad \text { and } \quad \sigma(t)=\min _{1 \leq i \leq 2}\left\{\sigma_{i}(t)\right\}=\sigma_{1}(t) .
$$

Observe, that the function $F_{\ell}: \mathbb{R}_{0} \rightarrow \mathbb{R}_{+}$defined as

$$
F_{\ell}(t)=\int_{t}^{\rho(t)} Q(s) \exp \left(\int_{\rho(t)}^{\sigma(s)} Q(u) \exp \left(\int_{u}^{\sigma(u)} R_{\ell}(\xi) d \xi\right) d u\right) d s
$$

attains its minimum at $t=7 k+3 / 4, k \in \mathbb{N}_{0}$, for every $\ell \in \mathbb{N}$. Specifically, by using an algorithm on MATLAB software and taking into account the fact that $Q(t)=$ $\sum_{i=1}^{2} q_{i}(t)=0.2$, we obtain

$$
F_{1}(t=7 k+3 / 4)=\int_{7 k+3 / 4}^{7 k+4} Q(s) \exp \left(\int_{7 k+4}^{\sigma(s)} Q(u) \exp \left(\int_{u}^{\sigma(u)} R_{1}(\xi) d \xi\right) d u\right) d s
$$


and therefore

$$
\limsup _{t \rightarrow \infty} F_{1}(t) \simeq 1.01496>1 .
$$

That is, condition (2.37) of Theorem 6 is satisfied for $\ell=1$, and therefore all solutions of (3.2) oscillate.

Observe, however, that

$$
\begin{aligned}
\limsup _{t \rightarrow \infty} \int_{t}^{\rho(t)} \sum_{i=1}^{2} q_{i}(s) d s=\limsup _{k \rightarrow \infty} \int_{7 k+3 / 4}^{7 k+4} 0.2 d s=0.65<1, \\
\liminf _{t \rightarrow \infty} \int_{t}^{\sigma(t)} \sum_{i=1}^{m} q_{i}(s) d s=\liminf _{k \rightarrow \infty} \int_{7 k+3}^{7 k+4} 0.2 d s=0.2<\frac{1}{e}, \\
=\liminf _{t \rightarrow \infty}\left[\frac{3}{20}\left(\sigma_{1}(t)-t\right)+\frac{1}{20}\left(\sigma_{1}(t)+0.5-t\right)\right] \\
=\liminf _{t \rightarrow \infty}\left[0.2\left(\sigma_{1}(t)-t\right)+0.025\right] \\
=\liminf _{t \rightarrow \infty}\left[0.2\left(\sigma_{1}(t)-t\right)\right]+0.025 \\
=0.2 \cdot 1+0.025=0.225<\frac{1}{e} .
\end{aligned}
$$

Also, observe that the function $W_{r}:[0, \infty) \rightarrow \mathbb{R}_{+}$defined as

$$
W_{r}(t)=\int_{t}^{\rho(t)} \sum_{i=1}^{2} q_{i}(\zeta) b_{r}\left(\rho(t), \sigma_{i}(\zeta)\right) d \zeta
$$

attains its maximum at $t=7 k+3 / 4$ and its minimum at $t=7 k+3, k \in \mathbb{N}_{0}$, for every $r \in \mathbb{N}$. Specifically,

$$
\begin{aligned}
W_{1}(t=7 k+3 / 4)= & \int_{7 k+3 / 4}^{7 k+4}\left[q_{1}(\zeta) b_{1}\left(7 k+4, \sigma_{1}(\zeta)\right)+q_{2}(\zeta) b_{1}\left(7 k+4, \sigma_{2}(\zeta)\right)\right] d \zeta \\
= & \int_{7 k+3 / 4}^{7 k+1}\left[q_{1}(\zeta) b_{1}\left(7 k+4, \sigma_{1}(\zeta)\right)+q_{2}(\zeta) b_{1}\left(7 k+4, \sigma_{2}(\zeta)\right)\right] d \zeta \\
& +\int_{7 k+1}^{7 k+2}\left[q_{1}(\zeta) b_{1}\left(7 k+4, \sigma_{1}(\zeta)\right)+q_{2}(\zeta) b_{1}\left(7 k+4, \sigma_{2}(\zeta)\right)\right] d \zeta \\
& +\int_{7 k+2}^{7 k+3}\left[q_{1}(\zeta) b_{1}\left(7 k+4, \sigma_{1}(\zeta)\right)+q_{2}(\zeta) b_{1}\left(7 k+4, \sigma_{2}(\zeta)\right)\right] d \zeta \\
& +\int_{7 k+3}^{7 k+4}\left[q_{1}(\zeta) b_{1}\left(7 k+4, \sigma_{1}(\zeta)\right)+q_{2}(\zeta) b_{1}\left(7 k+4, \sigma_{2}(\zeta)\right)\right] d \zeta \\
\simeq \quad & 0.7705 \\
W_{1}(t=7 k+3)=\int_{7 k+3}^{7 k+4} & {\left[q_{1}(\zeta) b_{1}\left(7 k+4, \sigma_{1}(\zeta)\right)+q_{2}(\zeta) b_{1}\left(7 k+4, \sigma_{2}(\zeta)\right)\right] d \zeta \simeq 0.28125 }
\end{aligned}
$$


Thus

$$
\begin{gathered}
\lim \sup _{t \rightarrow \infty} W_{1}(t) \simeq 0.7705<1 \\
\liminf t \rightarrow \infty \\
W_{1}(t) \simeq 0.28125<1 / e
\end{gathered}
$$

and

$$
0.7705<1-D(\beta) \simeq 0.9742 .
$$

Finally, by using an algorithm on MATLAB software, we obtain

$$
\begin{gathered}
\limsup _{t \rightarrow \infty} \int_{t}^{\rho(t)} \bar{Q}(s) \exp \left(\int_{\rho(t)}^{\sigma(s)} \bar{Q}_{1}(u) d u\right) d s \simeq 0.8687<1, \\
\quad 0.8687<1-D(\beta) \simeq 0.9742, \\
\limsup _{t \rightarrow \infty} \int_{t}^{\rho(t)} \bar{Q}(s) \exp \left(\int_{t}^{\sigma(s)} \bar{Q}_{1}(u) d u\right) d s \simeq 3.7953<\frac{1}{D(\beta)} \simeq 38.71,
\end{gathered}
$$

where $\lambda_{0}=1.29586$ is the smaller root of $e^{\lambda \beta}=\lambda$.

$$
\limsup _{t \rightarrow \infty} \int_{t}^{\rho(t)} \bar{Q}(s) \exp \left(\int_{\rho(s)}^{\sigma(s)} \bar{Q}_{1}(u) d u\right) d s \simeq 0.7234<\frac{1+\ln \lambda_{0}}{\lambda_{0}}-D(\beta) \simeq 0.9458
$$

and

$$
\liminf _{t \rightarrow \infty} \int_{t}^{\rho(t)} \bar{Q}(s) \exp \left(\int_{\rho(s)}^{\sigma(s)} \bar{Q}_{1}(u) d u\right) d s \simeq 0.2144<\frac{1}{e} .
$$

That is, none of the conditions (1.17), (1.18), (1.19), (1.22) (for $r=1$ ), (1.23) (for $r=1),(1.24)$ (for $r=1),(1.25)$ (for $j=1),(1.26)($ for $j=1),(1.27)$ (for $j=1$ ), (1.28) (for $j=1$ ) and (1.29) (for $j=1$ ) is satisfied.

Notation. It is worth noting that the improvement of condition (2.37) to the corresponding condition (1.17) is significant, approximately $56.14 \%$, if we compare the values on the left-side of these conditions. Also, the improvement compared to conditions (1.22) and (1.25) is very satisfactory, around $31.73 \%$ and $16.84 \%$, respectively. In addition, observe that conditions (1.22)-(1.24) and (1.25)-(1.29) do not lead to oscillation for first iteration. On the contrary, condition (2.37) is satisfied from the first iteration. This means that our condition is better and much faster than $(1.22)-(1.24)$ and $(1.25)-(1.29)$.

Remark 17. Similarly, one can construct examples to illustrate the other main results.

\section{REFERENCES}

[1] E. Braverman, G. E. Chatzarakis and I. P. Stavroulakis, Iterative oscillation tests for differential equations with several non-monotone arguments, Adv. Difference Equ., 2016, Paper No. 87, 18pp. 
[2] G.E. Chatzarakis, Differential equations with non-monotone arguments: Iterative Oscillation results, J. Math. Comput. Sci., 6 (2016), No. 5, 953-964.

[3] G.E. Chatzarakis and H. Péics, Differential equations with several non-monotone arguments: An oscillation result, Appl. Math. Lett., 68 (2017), 20-26.

[4] G. E. Chatzarakis, Oscillations caused by several non-monotone deviating arguments, Differ. Equ. Appl., 9 (2017), no. 3, 285-310.

[5] L. H. Erbe, Qingkai Kong and B.G. Zhang, Oscillation Theory for Functional Differential Equations, Marcel Dekker, New York, 1995.

[6] L. H. Erbe and B. G. Zhang, Oscillation of first order linear differential equations with deviating arguments, Differential Integral Equations, 1 (1988), 305-314.

[7] N. Fukagai and T. Kusano, Oscillation theory of first order functional-differential equations with deviating arguments, Ann. Mat. Pura Appl. 136 (1984), 95-117.

[8] M.K. Grammatikopoulos and M. R.S. Kulenovic, First Order Functional Differential Inequalities with Oscillating Coefficients, Nonlinear Analysis TMA 8 (9), (1984), 1043-1054.

[9] B. R. Hunt and J. A. Yorke, When all solutions of $x^{\prime}(t)=-\sum q_{i}(t) x\left(t-T_{i}(t)\right)$ oscillate, J. Differential Equations 53 (1984), 139-145.

[10] J. Jaroš and I. P. Stavroulakis, Oscillation tests for delay equations, Rocky Mountain J. Math., 45 (2000), 2989-2997.

[11] M. Kon, Y. G. Sficas and I. P. Stavroulakis, Oscillation criteria for delay equations, Proc. Amer. Math. Soc., 128 (1994), 675-685.

[12] R. G. Koplatadze and T. A. Chanturija, Oscillating and monotone solutions of first-order differential equations with deviating argument, (Russian), DifferentsialınyeUravneniya 18 (1982), 1463-1465, 1472.

[13] R.G. Koplatadze and G. Kvinikadze, On the oscillation of solutions of first order delay differential inequalities and equations, Georgian Math. J., 3 (1994), $675-685$.

[14] T. Kusano, On even-order functional-differential equations with advanced and retarded arguments, J. Differential Equations 45 (1982), 75-84.

[15] M. K. Kwong, Oscillation of first-order delay equations, J. Math. Anal. Appl. 156 (1991), 274-286.

[16] G. Ladas, V. Lakshmikantham and L. S. Papadakis, Oscillations of higher-order retarded differential equations generated by the retarded arguments, Delay and Functional Differential Equations and their Applications, Academic Press, New York, 1972, 219-231. 
[17] G. Ladas and I. P. Stavroulakis, Oscillations caused by several retarded and advanced arguments, J. Differential Equations 44 (1982), 134-152.

[18] G.S. Ladde, Oscillations caused by retarded perturbations of first order linear ordinary differential equations, Atti Acad. Naz. Lincei Rendiconti 63 (1978), $351-359$.

[19] G.S. Ladde, V. Lakshmikantham, B.G. Zhang, Oscillation Theory of Differential Equations with Deviating Arguments, Monographs and Textbooks in Pure and Applied Mathematics, vol. 110, Marcel Dekker, Inc., New York, 1987.

[20] X. Li and D. Zhu, Oscillation and nonoscillation of advanced differential equations with variable coefficients, J. Math. Anal. Appl. 269 (2002), 462-488.

[21] A. D. Myshkis, Linear homogeneous differential equations of first order with deviating arguments, Uspekhi Mat. Nauk, 5 (1950), 160-162 (Russian).

[22] J. S. Yu, Z. C. Wang, B. G. Zhang and X. Z. Qian, Oscillations of differential equations with deviating arguments, Panamer. Math. J. 2 (1992), no. 2, 59-78.

[23] B. G. Zhang, Oscillation of solutions of the first-order advanced type differential equations, Science exploration 2 (1982), 79-82.

[24] D. Zhou, On some problems on oscillation of functional differential equations of first order, J. Shandong University 25 (1990), 434-442. 